\title{
One-Dimensional Disordered Bosonic Hubbard Model: A Density-Matrix Renormalization Group Study
}

\author{
Ramesh V. Pai, ${ }^{1}$ Rahul Pandit,,${ }^{1,2}$ H. R. Krishnamurthy, ${ }^{1,2}$ and S. Ramasesha ${ }^{1,3}$ \\ ${ }^{1}$ Jawaharlal Nehru Centre for Advanced Scientific Research, Bangalore 560 064, India \\ ${ }^{2}$ Department of Physics, Indian Institute of Science, Bangalore 560 012, India \\ ${ }^{3}$ Solid State and Structural Chemistry Unit, Indian Institute of Science, Bangalore 560 012, India
}

\begin{abstract}
We use the density-matrix renormalization group to study the one-dimensional bosonic Hubbard model, with and without disorder. We obtain the gaps in all phases, certain correlation functions, and the superfluid density. A finite-size-scaling analysis enables us to obtain an accurate phase diagram and the $\beta$ function at the superfluid-Mott insulator transition and the Kosterlitz-Thouless essential singularity in the pure case. We also obtain coupling constants used in effective field theories for this system.
\end{abstract}

There has been a renaissance in the study of quantum phase transitions in interacting disordered Bose systems in recent years. In particular, experiments on ${ }^{4} \mathrm{He}$ adsorbed in porous media [1] and disordered superconducting films [2] motivated the study of transitions between superfluid, Bose glass, and (bosonic) Mott insulator phases. Also type-II superconductors with columnar defects may show analogs of these phases [3]. The disordered, bosonic Hubbard model is the simplest model which has these three phases, so it has been studied extensively [4-7]. In particular, numerical simulations [5] of this model have been more successful than those of its fermionic analogs for reasons such as the absence of a Monte Carlo sign problem for bosons. However, in dimension $d=1$, the pure fermionic Hubbard model can be solved exactly at temperature $T=0$ by the Bethe ansatz unlike its bosonic analog [8]. It is interesting, therefore, to study the $d=1$ disordered, bosonic Hubbard model at $T=0$ by the best approximate method available for such purposes, namely, the density-matrix renormalization group [9]. In this paper we present such a study. Perhaps this is the most complex model to which the densitymatrix renormalization group has been applied so far. So, apart from the intrinsic interest of the model, it provides a good testing ground for this technique.

The $d=1$ bosonic Hubbard Hamiltonian is

$$
\frac{\mathcal{H}}{2 t}=-\frac{1}{2} \sum_{\langle i j\rangle} a_{i}^{\dagger} a_{j}+\frac{U}{2} \sum_{i} n_{i}\left(\hat{n}_{i}-1\right)-\sum_{i} \mu_{i} \hat{n}_{i}
$$

where $-t$ is the hopping amplitude between nearestneighbor pairs of sites $\langle i j\rangle, U$ the on-site repulsive energy, $a_{i}^{\dagger}\left(a_{i}\right)$ the boson creation (annihilation) operator at site $i$, and $\hat{n}_{i} \equiv a_{i}^{\dagger} a_{i}$. The on-site chemical potential $\mu_{i}$ is a random number distributed uniformly between $-\Delta$ and $\Delta$, i.e., $\Delta=0$ for the pure case. We work in the canonical ensemble with a fixed boson density $\rho$ and set the energy scale by choosing $2 t=1$. Model (1) has qualitatively different behaviors for integer and noninteger $\rho$; e.g., the Mott insulator phase occurs only if $\rho$ is an integer [4]. Thus for integer $\rho$ we expect Bose glass-superfluid, superfluid-Mott insulator, and Mott insulator-Bose glass transitions but only the first of these transitions for noninteger $\rho$. In this paper we concentrate on the integer case $\rho=1$ since this displays all these transitions.

Our density-matrix renormalization group for model (1) yields many interesting results. First in the pure case $(\Delta=$ 0 ), the superfluid phase should exhibit only quasi-longrange order in $d=1$ and $T=0$; and the superfluid-Mott insulator transition should be of the Kosterlitz-Thouless type $[4,10]$. We confirm this behavior via a direct calculation by computing the gap $G_{L}$ (Fig. 1) for different system sizes $L$ and couplings $U$. A plot of $L G_{L}$ versus $U$ (Fig. 2) shows that, for $U<U_{c} \simeq 1.7$, curves for different $L$ coalesce, indicating the power-law nature of the $d=1$ superfluid phase. By using Roomany-Wyld approximants [11] we obtain the $\beta$ function which shows the KosterlitzThouless essential singularity (inset of Fig. 2). We also obtain the universal jump in the superfluid density $\rho_{s}$ at the Kosterlitz-Thouless transition and the $1 / \ln (L)$ corrections

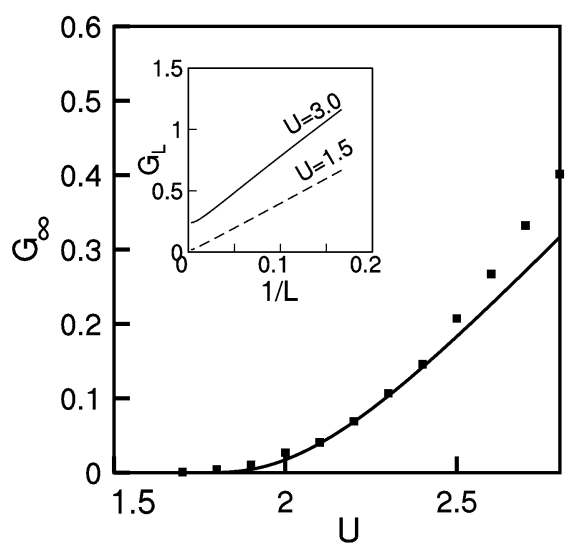

FIG 1. Squares show the gap $G_{\infty}$ vs $U$ in the Mott insulator phase. The solid line is $G(U)=7.5 \exp (-3.22 / \sqrt{U-1.7})$. Inset: $G_{L}$ vs $1 / L$ for $U=1.5$ and 3.0. 


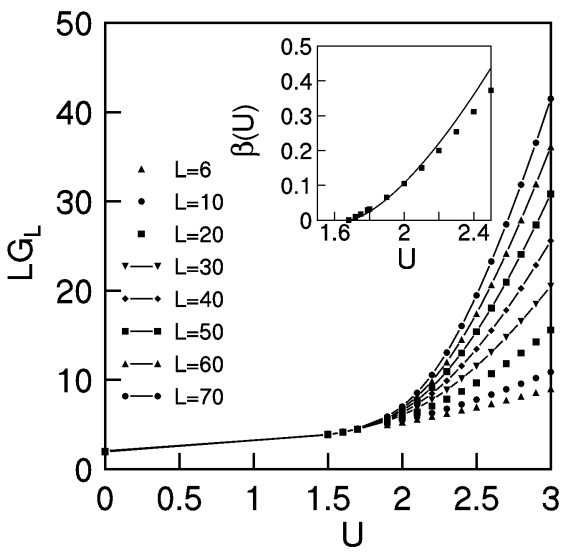

FIG 2. $L G_{L}$ vs $U$ showing the coalescence of the curves for different $L$ at the Kosterlitz-Thouless type Mott insulatorsuperfluid transition (see text). Inset: data for $\beta$ function (squares) and the fit (solid line) $\beta(U) \sim 0.59(U-1.68)^{1.5}$.

to $\rho_{s}(L)$ in the superfluid phase (Fig. 3) that are consistent with the presence of a marginal operator [12]. Next we identify parameters in an effective field theory for this transition obtained via bosonization [13] and show that the asymptotic behavior of the correlation function $\left\langle a_{i}^{\dagger} a_{j}\right\rangle$ is consistent with the prediction of this field theory. In the disordered case $(\Delta \neq 0)$, we use analogs of Fig. 2 to obtain the Mott insulator-superfluid, Mott insulator-Bose glass (Fig. 4), and the superfluid-Bose glass (Fig. 5) phase boundaries. Our data are consistent with a gapless Bose glass phase. From plots like Figs. 4 and 5, we obtain, for the first time, an accurate phase diagram (Fig. 6) of the $d=1$ disordered bosonic Hubbard model at $\rho=1$.

Our density-matrix renormalization group, done in the standard way [9], starts with a superblock $B_{2 L}$ of $2 L$ sites which consists of left $\left(B_{L}^{l}\right)$ and right $\left(B_{L}^{r}\right)$ blocks with $L$ sites each. $B_{L}^{l}$ and $B_{L}^{r}$ are not identical if $\Delta \neq 0$.

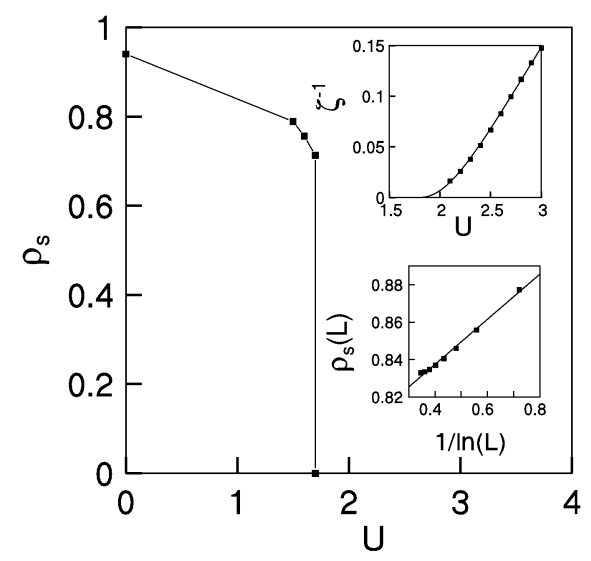

FIG 3. The universal jump in $\rho_{s}$ at the superfluid-Mott insulator transition. Top inset: data (squares) for $\zeta^{-1}(U)$ (see text) and the fit $\zeta^{-1}(U)=2.5 \exp (-3.22 / \sqrt{U-1.68}$ ) (solid line). Bottom inset: $\rho_{s}$ vs $1 / \ln (L)$ for $U=1.5$.

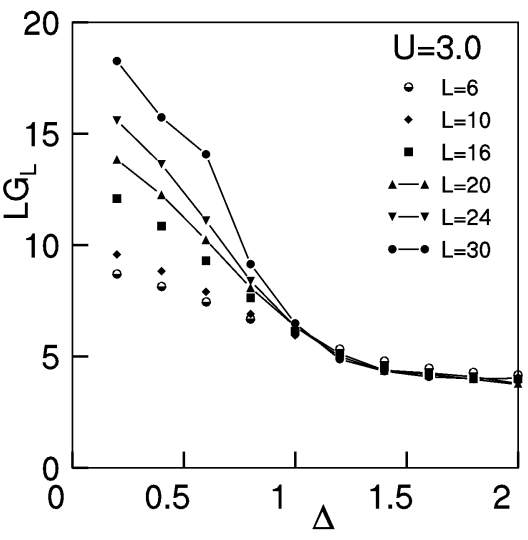

FIG 4. $L G_{L}$ vs $\Delta$ showing the coalescence of the curves for different $L$ at the Mott insulator-Bose glass transition.

We obtain the desired target state of the Hamiltonian for $B_{2 L}$ and use that to construct the reduced block density matrices for $B_{L}^{l}$ and $B_{L}^{r}[9,14]$. The target state is chosen to be the ground (first-excited) state of $B_{2 L}$ if we want the ground (first-excited) state of model (1). We diagonalize the block density matrices, retain as basis states $M$ eigenvectors (usually $M=64$, often checked against $M=96$ ) corresponding to the $M$ largest eigenvalues, and obtain matrix representations of operators (e.g., $\mathcal{H}$ ) for the sub-blocks $B_{L}^{l}$ and $B_{L}^{r}$ in this basis. We now add two sites to $B_{2 L}$ and repeat the above procedure by replacing the sub-blocks by $B_{L+1}^{l} \equiv B_{L}^{l} \bullet$ and $B_{L+1}^{r} \equiv$ $B_{L}^{r} \bullet$, where $\bullet$ represents the added site. This procedure is repeated until the energy per site converges to the desired accuracy [15]. For periodic $(p)$ and twisted $(\theta)$ boundary conditions we use the superblock configuration $B_{L}^{l} \bullet B_{L}^{r} \bullet$, whereas we use $B_{L}^{l} \bullet \bullet B_{L}^{r}$ for open $(o)$ boundary condition [9]. For $\theta$ boundary conditions we multiply $t$ by $e^{i \theta / 2}$ on the two bonds that connect the left and right sub-blocks; $\theta=0$ and $\theta=\pi$ yields periodic and antiperiodic $(a)$ boundary conditions, respectively. We diagonalize $n^{2} M^{2} \times n^{2} M^{2}$ Hamiltonian matrices at

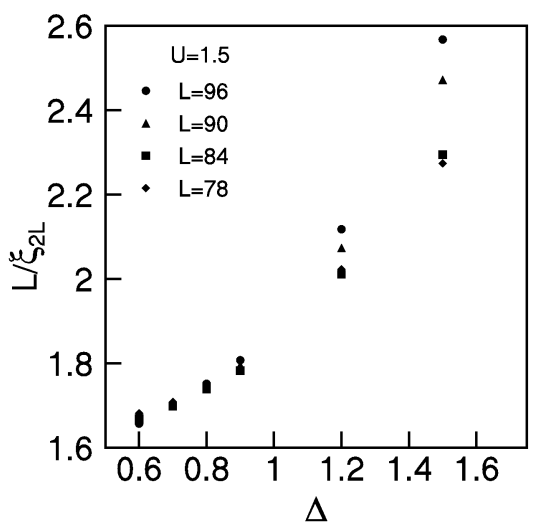

FIG 5. $L / \xi_{2 L}$ (see text) vs $\Delta$ showing the coalescence of the curves for different $L$ at the Bose glass-superfluid transition. 


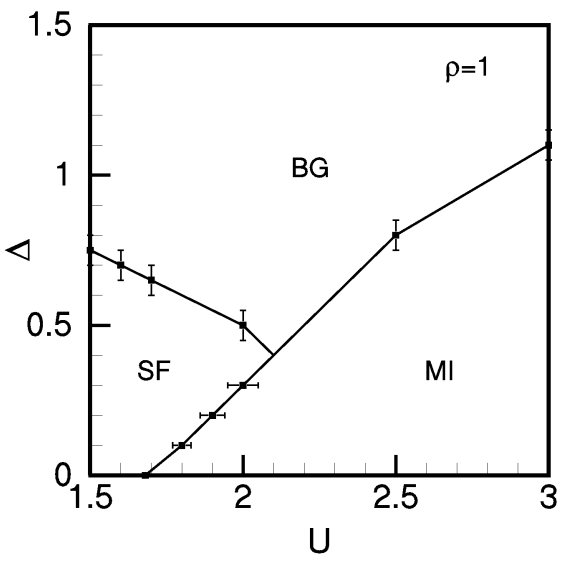

FIG 6. The $\rho=1$ phase diagram for model (1) showing superfluid (SF), Bose glass (BG), and Mott insulator (MI) phases. The phase boundaries have been drawn through the computed points (squares with error bars) to guide the eye. All transitions are continuous.

each density-matrix renormalization group step, where $n$ is the number of states per site. For bosons $n=\infty$, but in a practical calculation we must use a truncated basis. Clearly $n=2$ suffices in the hard-core limit $U=\infty$. For $U>1.5$ we find $n=4$; i.e., a truncated occupationnumber basis with $0,1,2$, or 3 bosons per site, works well. Our results are hardly modified when we use $n=5$ or 6 [14].

We denote the ground (first-excited) state energy of a chain of length $L$ by $E_{0}^{\alpha}(L)\left[E_{1}^{\alpha}(L)\right]$, with boundary condition $\alpha(=o, p, a, \theta)$. The gap $G_{L}$ is obtained most accurately [9] as $E_{1}^{o}(L)-E_{0}^{o}(L)$. To extract $\rho_{s}$ we use [16] $\rho_{s}(L)=\frac{2 L}{\pi^{2}}\left[E_{0}^{a}(L)-E_{0}^{p}(L)\right]$ or $\rho_{s}(L)=$ $\lim _{\theta \rightarrow 0} L \frac{\partial^{2} E_{0}^{\theta}(L)}{\partial \theta^{2}}$; these are equivalent in the thermodynamic limit $L \rightarrow \infty$, but we find that the second expression converges better. We also compute the correlation function $\Gamma_{L}(r)=\left\langle\psi_{0 L}^{o}\left|a_{i}^{\dagger} a_{i+r}\right| \psi_{0 L}^{o}\right\rangle$ and its second moment $\xi_{2 L}^{2} \equiv \sum_{r} r^{2} \Gamma_{L}(r) / \sum_{r} \Gamma_{L}(r)$, where $\left|\psi_{0 L}^{o}\right\rangle$ is the ground-state wave function for size $L$ with open boundary conditions.

We have tested our density-matrix renormalization group for $U=\infty, \rho=1 / 2$, and $\Delta=0$ when model (1) becomes the exactly solvable, spin- $\frac{1}{2} X Y$ model [17]. Our results (with $M=64$ and a final $L=50$ ) agree well with the exact ones: $E_{0}^{o} / L$ and $E_{0}^{p} / L$ are accurate to 8 and 4 figures, respectively (both must be equal in the limit $L \rightarrow \infty)$; and $\rho_{s}(L) \approx \frac{1}{\pi}+O\left(L^{-2}\right)[17]$.

Figure 1 shows $G_{L}$ for various values of $U$ with $\Delta=$ 0 . As $L \rightarrow \infty, G_{L} \rightarrow G_{\infty}>0$ for $U>1.7$, yielding the Mott insulator phase. A gapless superfluid phase appears for $U \leq 1.7$. The gap $G_{L} \sim \xi_{2 L}^{-1}$. If a KosterlitzThouless transition occurs at $U_{c}$ then [10], for $L=$ $\infty, \quad \xi_{2 \infty} \sim \exp \left(\frac{a}{\sqrt{U-U_{c}}}\right)$. Our data are consistent with this (Fig. 1) and yield $U_{c} \simeq 1.7$; this estimate can be improved [11]: A plot of $L G_{L}$ vs $U$ should show curves for different $L$ crossing at the critical point. Our data (Fig. 2) show that not only do these curves come together at $U_{c} \simeq 1.7$, but they coalesce after that; i.e., the low- $U$ phase is itself critical [11]. These data can be used with a phenomenological renormalization group [11] to extract the $\beta$ function $\lim _{b \rightarrow 1} \partial(U) / \partial(b)=\partial \ln (G) / \partial(U)$, where $b=1+\delta b$ is the scale factor. It is convenient to use the Roomany-Wyld approximants [11]

$$
\beta_{L L^{\prime}}^{\mathrm{RW}}(U)=\frac{1+\ln \left(G_{L} / G_{L^{\prime}}\right) / \ln \left(L / L^{\prime}\right)}{\left(G_{L}^{\prime} G_{L^{\prime}}^{\prime} / G_{L} G_{L^{\prime}}\right)^{1 / 2}},
$$

where $G_{L}^{\prime} \equiv \partial G(L) / \partial U . \quad$ As $L, L^{\prime} \rightarrow \infty, \quad \beta_{L L^{\prime}}^{\mathrm{RW}}(U) \rightarrow$ $\beta(U)$. At a Kosterlitz-Thouless transition $\beta(U) \approx$ $\frac{2}{a}\left(U-U_{c}\right)^{1.5}$. Our $\beta$ function [obtained with $L=20$ and $L^{\prime}=22$ in Eq. (2)] is consistent with this behavior (Fig. 2 inset) with $U_{c}=1.68 \pm 0.01$ and $a=0.59 \pm 0.05$. We also expect a universal jump in $\rho_{s}$ across the superfluid-Mott insulator transition. This behavior (Fig. 3) is not easy to extract numerically but follows thus: In the Mott insulator phase we find $\rho_{s}(L) \sim \exp \left[-L \zeta^{-1}(U)\right]$, with $\zeta(U) \sim \xi_{2 L}(U)$. By computing $\rho_{s}(L)$ for $U>U_{c}=1.68$, we have obtained (top inset of Fig. 3) $\zeta^{-1} \sim \exp \left(\frac{-a}{\sqrt{U-U_{c}}}\right)$, whence $\rho_{s}(\infty)=0$ for $U>U_{c}$ [18]. In the superfluid phase we find (bottom inset of Fig. 3) $\rho_{s}(L) \approx \rho_{s}(\infty)+C / \ln L$, where $C$ depends on $U$; we extract $\rho_{s}(L=\infty)$ from such a fit. The $(\ln L)^{-1}$ term is consistent with the presence of a marginal operator [12] in the superfluid phase. We find

$$
\Gamma(r) \sim r^{-K(U) / 2}
$$

for $U<U_{c}$ with $K$ an exponent that depends on $U$ (Table I) as expected for a Kosterlitz-Thouless transition [10]. For $U>U_{c}, \Gamma(r)$ decays exponentially.

In $d=1$ interacting boson problems like model (1) are often studied [13] via an effective Hamiltonian $\mathcal{H}_{\text {eff }}$ for long-wavelength and low-energy properties. For integer filling (say, $\rho=1$ )

$$
\begin{gathered}
\mathcal{H}_{\text {eff }}=\frac{1}{2 \pi} \int d x\left(\frac{u}{K}(\pi \Pi)^{2}+u K \partial_{x}\left[\phi^{2}(x)\right]\right. \\
+\cos [2 \phi(x)])
\end{gathered}
$$

where $\Pi$ and $\phi$ are canonically conjugate bosonic fields, $u$ the phase velocity of sound waves, and $K$ is given by Eq. (3). A renormalization group study [13] of $\mathcal{H}_{\text {eff }}$

TABLE I. The field theory coupling constants $u$ and $K$ (see text) for $U=1.5,1.6$, and $U=U_{c}=1.68$.

\begin{tabular}{lccc}
\hline \hline$U$ & $u$ & $K=u / \pi \rho_{s}$ & $K$ from Eq. (3) \\
\hline 1.5 & 0.789 & $0.459 \pm 0.003$ & $0.464 \pm 0.003$ \\
1.6 & 0.756 & $0.493 \pm 0.003$ & $0.482 \pm 0.005$ \\
1.68 & 0.723 & $0.525 \pm 0.004$ & $0.521 \pm 0.007$ \\
\hline \hline
\end{tabular}


predicts a superfluid-Mott insulator transition at $K\left(U_{c}\right)=$ $1 / 2$. Our density-matrix renormalization group study allows us to compute $u$ and $K$ as functions of $U$ in model (1) (Table I) via the conformal-invariance result [19] $E_{0}^{p}(L)=L E_{0}(L=\infty)-\frac{\pi}{6} \frac{u c}{L}+\cdots$, and $\rho_{s}=u / \pi K$, where the central charge $c=1$. Table I indicates that the $K$ 's determined from $\rho_{s}$ (column 3) and $\Gamma(r)$ (column 4) agree more or less given our error bars [20].

In the disordered case, for small $\Delta(\leq 0.4)$, the Mott insulator-superfluid transition survives, but with a $U_{c}$ that increases with $\Delta$. This is obtained by a careful finitesize scaling analysis of $G_{L}$ and $\rho_{s}(L)$. For larger values of $\Delta$, we obtain the Mott insulator-Bose glass transition by studying the $L$ and $\Delta$ dependence of $G_{L}$ (Fig. 4, for $U=3.0$ ). These data are consistent with a transition to a gapless Bose glass phase: Plots of $L G_{L}$ vs $\Delta$ for different values of $L$ come together at a point $\Delta_{c}(\simeq 1.1$ for $U=3.0$ ) and coalesce after that. However, in spite of averaging (over 10-25 realizations of $\mu_{i}$ ), these data are not good enough to obtain the $\beta$ function and critical exponents at this transition. We obtain the superfluidBose glass boundary by studying the $L$ and $\Delta$ dependence of $\xi_{2 L}$ (Fig. 5, for $U=1.5$ ). The coalescence of these curves below $\Delta_{c}$ shows that $\Gamma(r)$ decays exponentially in the Bose glass phase but algebraically in the superfluid phase. Data extracted from plots like Figs. 4 and 5 lead to our phase diagram (Fig. 6) for model (1) at the integer filling $\rho=1$. Some workers [4] have suggested that the Bose glass phase might always separate the Mott insulator and superfluid phases. Our phase diagram (Fig. 6) shows that if this is the case, then the region of Bose glass separating superfluid and Mott insulator phases must be very narrow indeed.

We thank S. Dhar, T. Giamarchi, S. Pati, D. Sen, and B.S. Shastry for discussions, UGC (India) for support, and SERC (IISc, Bangalore) for computer resources. One of us (R.P.) thanks the Department of Physics, University of Missouri-Columbia for hospitality where a part of this paper was written.

[1] M. H. W. Chan, K. I. Blum, S. Q. Murphy, G. K. S. Wong, and J. D. Reppy, Phys. Rev. Lett. 61, 1950 (1988).

[2] D. B. Haviland, Y. Liu, and A. M. Goldman, Phys. Rev. Lett. 62, 2180 (1989).

[3] D. R. Nelson and V.M. Vinokur, Phys. Rev. B 48, 13060 (1993); G. Blatter, M. V. Feigelmann, V. B. Geshkenbein, A. I. Larkin, and V. M. Vinokur, Rev. Mod. Phys. 66, 1125 (1994).

[4] M.P.A. Fisher, P. B. Weichmann, G. Grinstein, and D. S. Fisher, Phys. Rev. B 40, 546 (1989); M. Ma, B. I. Halperin, and P. A. Lee, ibid. 34, 3136 (1986).

[5] R. T. Scalettar, G. G. Batrouni, and G. T. Zimanyi, Phys. Rev. Lett. 66, 3144 (1991); W. Krauth and
N. Trivedi, Europhys. Lett. 14, 627 (1991); M. Makivić, N. Trivedi, and S. Ullah, Phys. Rev. Lett. 71, 2307 (1993); N. Trivedi and M. Makivić, ibid. 74, 1039 (1995); S. Zhang, N. Kawashima, J. Carlson, and J. E. Gubernatis, ibid. 74, 1500 (1995); M. Wallin, E. S. Sorensen, S. M. Girvin, and A. P. Young, Phys. Rev. B 49, 12115 (1994).

[6] K. Sheshadri, H. R. Krishnamurthy, R. Pandit, and T. V. Ramakrishnan, Europhys. Lett. 22, 257 (1993); Phys Rev. Lett. 75, 4075 (1995); P. Nissamoniphong, L. Zhang, and M. Ma, ibid. 71, 3830 (1993).

[7] T. Giamarchi and H. J. Schultz, Europhys. Lett. 3, 1287 (1987); Phys. Rev. B 37, 325 (1988); K. G. Singh and D. S. Rokhsar, ibid. 46, 3002 (1992).

[8] F. D. M. Haldane, Phys. Lett. 80A, 281 (1980); T. C. Choy and F. D. M. Haldane, ibid. 90A, 83 (1982).

[9] S. R. White, Phys. Rev. Lett. 69, 2863 (1992); Phys. Rev. B 48, 10345 (1993).

[10] J. M. Kosterlitz and D. J. Thouless, J. Phys. C 6, 1181 (1973).

[11] M. N. Barber, in Phase Transitions and Critical Phenomena, edited by C. Domb and J. L. Lebowitz (Academic, New York, 1990), Vol. 8, p. 145; H.H. Roomany and H. W. Wyld, Phys. Rev. D 21, 3341 (1980).

[12] I. Affleck, D. Gepner, H. J. Schulz, and T. Ziman, J. Phys. A 22, 511 (1989).

[13] F. D. M. Haldane, Phys. Rev. Lett. 45, 1358 (1980); T. Giamarchi, Phys. Rev. B 46, 342 (1992).

[14] R. V. Pai, R. Pandit, H. R. Krishnamurthy, and S. Ramasesha (to be published).

[15] The truncation error $\left(\equiv 1-\sum_{m=1}^{M} w_{m}\right.$, with $w_{m}$ the eigenvalues of the density matrix) was $\simeq 10^{-6}$ for $\Delta=0$ and $\simeq 10^{-4}$ for $\Delta=1.5$.

[16] M. E. Fisher, M. N. Barber, and D. Jasnow, Phys. Rev. A 8, 1111 (1973); J. Banavar and D. Jasnow, J. Phys. A 11, 1361 (1978).

[17] E. Lieb, T. Schultz, and D. Mattis, Ann. Phys. (N.Y.) 16, 407 (1961); for $\rho=1 / 2, \Delta=0$, and $U=\infty, \rho_{s}(\infty)-$ $\rho_{s}(L) \sim L^{-2}$, we have a free-fermion problem and no marginal operator.

[18] $\zeta$ diverges exponentially as $U \downarrow U_{c}$, and our densitymatrix renormalization group results for periodic boundary conditions develop errors for $L>40$, so we cannot fit the exponential decay of $\rho_{s}(L)$ with $L$ for $1.7<U<2.1$. However, our data for $U \geq 2.1$ lead to the fit shown in the top inset of Fig. 3 with a $U_{c}$ that is consistent with the value we get from our $\beta$ function. This also prevents us from seeing the discontinuous jump of $\rho_{s}$ directly, but it can be surmised as described in the text.

[19] J. Cardy, in Field, Strings and Critical Phenomena, Proceedings of the Les Houches Summer School, Session XLIX, edited by E. Brezin and J. Zinn-Jutin (NorthHolland, Amsterdam, 1990), p. 169.

[20] Only the fitting errors are shown. Systematic errors caused, e.g., by stopping the density-matrix renormalization group at some finite $L$ account, we believe, for the remaining discrepancies and for $K$ not being exactly 0.5 at the transition. 